\title{
CONF-800932--4
}

GA-A15984

\section{LOWER HYBRID WAVE ELECTRON HEATING EXPERIMENTS IN DOUBLET IIA}

\author{
by \\ R. L. FREEMAN, J. L. LUXON, V. S. CHAN, S. C. CHIU, \\ J. C. DEBOO, R. W. HARVEY, T. H. JENSEN, R. J. LAHAYE, \\ J. M. LOHR, C. P. MOELLER, T. OHKAWA, J. C. RIORDAN, \\ J. F. TOOKER, and D. F. VASLOW
}

JULY 1980 


\section{DISCLAIMER}

This report was prepared as an account of work sponsored by an agency of the United States Government. Neither the United States Government nor any agency Thereof, nor any of their employees, makes any warranty, express or implied, or assumes any legal liability or responsibility for the accuracy, completeness, or usefulness of any information, apparatus, product, or process disclosed, or represents that its use would not infringe privately owned rights. Reference herein to any specific commercial product, process, or service by trade name, trademark, manufacturer, or otherwise does not necessarily constitute or imply its endorsement, recommendation, or favoring by the United States Government or any agency thereof. The views and opinions of authors expressed herein do not necessarily state or reflect those of the United States Government or any agency thereof. 


\section{DISCLAIMER}

Portions of this document may be illegible in electronic image products. Images are produced from the best available original document. 


\section{DISCLAIMER}

This report was prepared as an account of work sponsored by an agency of the United States Government. Neither the United States Government nor any agency thereof, nor any of their employees, makes any warranty, express or implied, or assumes any legal liability or responsibility for the accuracy, completeness, or usefulness of any information, apparatus, product, or process disclosed, or represents that its use would not infringe privately owned rights. Reference herein to any specific commercial product, process, or service by trade name, trademark, manufacturer, or otherwise, does not necessarily constitute or imply its endorsement, recommendation, or favoring by the United States Government or any agency thereof. The views and opinions of authors expressed herein do not necessarily state or reflect those of the United States Government or any agency thereof. 
GA-A15984

DiSCLAIMER This book was prepared as an account of work sponsond by hat an of their employees, makes any Neither the United States Government nor any asence liability or responsibility for the accursey.

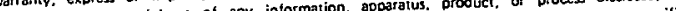

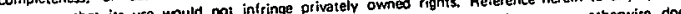

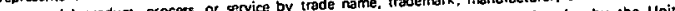

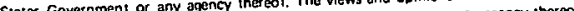

\title{
LOWER HYBRID WAVE ELECTRON HEATING EXPERIMENTS IN DOUBLET IIA
}

by

R. L. FREEMAN, J. L. LUXON, V. S. CHAN, S. C. CHIU, J. C. DEBOO, R. W. HARVEY, T. H. JENSEN, R. J. LAHAYE, J. M. LOHR, C. P. MOELLER, T. OHKAWA, J. C. RIORDAN*, J. F. TOOKER, and D. F. VASLOW

This is a preprint of a paper to be presented at the Joint Varenna-Grenoble International Symposium on Heating in Toroidal Plasmas, September 3-12, 1980, Como, Italy.

\author{
Work supported by \\ Department of Energy \\ Contract DE-AT03-76ET51011
}

*Present Address: Maxwell Labs Inc. San Diego, California

GENERAL ATOMIC PROJECT 3235

JULY 1980

\section{GENERAL ATOMIC COMPANY}


LOWER HYBRID WAVE ELECTRON HEATING EXPERIMENTS

IN DOUBLET IIA**

by

R.L. Freeman, J.L. Luxon, V.S. Chan, S.C. Chiu, J.C. DeBoo, R.W. Harvey T.H. Jensen, R.J, LaHaye J.M. Lohr, C.P. Moeller, T. Ohkawa, J.C. Riordan*, J.F. Tooker, and D.F. Vaslow

General Atomic Company, San Diego, CA.

\begin{abstract}
Experiments designed to heat electrons by Landau damping of waves at approximately twice the lower hybrid frequency have been carried out on Doublet IIA. This objective is in contrast to other lower hybrid experiments which are designed to heat ions using frequencies corresponding to the lower hybrid resonance frequency. Up to $500 \mathrm{~kW}$ of rf power was applied to discharge with approximately $100 \mathrm{~kW}$ ohmic input using parallel wavelengths chosen to optimize the spatial distribution of the power deposition based on linear or quasi-linear Landau damping.

Coupling of the power to both electrons and ions was observed, but there was no indication of effective bulk heating of either species. The desired slow wave propagated into the plasma and efficient coupling of the wave energy to the plasma occured, but this energy was poorly confined. Two possible models of the absorption and loss mechanisms remain unresolved 1 ) The power is coupled to energetic electrons via Landau damping and is lost via anomolous electron transport before it can thermalize. 2) The power is coupled to energetic ions and is lost by direct particle losses before it can thermalize.
\end{abstract}

* Present address: Maxwell Labs, San Diego, CA 


\section{INTRODUCTION}

This paper summarizes the results of experiments on Doublet IIA to evaluate the use of electron Landau damping of lower hybrid waves as an auxiliary heating method for tokamaks. Ample auxiliary power was available; over $500 \mathrm{kw}$ of radio frequency (rf) power at $800 \mathrm{MHz}$ and $915 \mathrm{MHz}$ was applied to circular tokamak discharges whereas the plasma was sustained by approximately $100 \mathrm{kw}$ of ohmic power. The lower hybrid wavelength, characterized by the parallel index of refraction $\left(\mathrm{n}_{\|}=c \mathrm{k}_{\|} / \omega\right)$ was chosen so that that wave-particle resonance occurs in the tail of the electron distribution. The rf power levels are sufficient to expect a quasi-linear plateau in the electron velocity distribution, and/or non-linear mechanisms such as electron trapping in the waves and parametric decays. Furthermore the electron energy confinement time is sufficient to expect that rf energy deposited in the tail of the electron distribution will be transferred via collisions to the thermal electrons..

The primary results from this experiment are that the rf power is coupled to the plasma and transported into the interior of the plasma where the wave energy was then transferred to the plasma particles. This energy was ultimately transported to the plasma edge via particle transport. There was only sporadic evidence of increases in the central electron bulk temperature and no heating of the thermal ions. 
Despite the efficient transfer of the wave energy to the charged particles, rapid loss of this energy prevents its thermalization. Whether the energy transfer to the plasma edge was by electrons or ions has not been definitely ascertained.

The experimental data is rich in physical phenomena, but the discussion here will be limited to only those features essential for understanding the power balance. A paper covering most of the detailed observations in the experiments is being prepared for publication (reference 1).

The basic device, plasma parameters, and wave coupling are discribed briefly in Section II. The experimental observations of the effect of the rf power on the plasma are discribed in Section III and the results are summarized in Section IV. 


\section{EXPERIMENTAL ARRANGEMENT}

The basic plasma and device parameters are shown in Table 1 , and the reader is referred to references 1 and 2 for more details of the Doublet IIA device. These experiments were carried out on circular discharges to minimize the ohmic input and simplify the control of the discharge.

Due to access restrictions and the need for large values of $n_{\|}$. slow wave structures, each capable of coupling $100 \mathrm{~kW}$ to the plasma, were utilized in these experiments rather than the more commonly used phased waveguide arrays. The slow wave structures are positioned at the top and bottom of the plasma cross-section as shown in Figure 1 . The top view of Doublet IIA illustrates the toroidal extent of the structures. Details of the construction and operation of the slow wave structure are discussed in reference 3 . Most of the structures $\left(n_{\|}=11,14,16,19,22,28\right)$ were designed to operate at $800 \mathrm{MHz}$ with the exception of one set at $n_{\|}=16$ designed for $915 \mathrm{MHz}$. In the rest of this paper the $n_{\|}=16,915 \mathrm{MHz}$ antennas will be labelled $n_{\|}=16^{*}$ to distinguish them from the $800 \mathrm{MHZ}$ antennas. Six slow wave structures could be accomodated in the machine. 
Reference 1 also discusses $r f$ probe measurements inside the plasma which demonstrated the existence of resonance cones in the plasma at the location predicted theoretically for the launched waves. The wave phase velocity was also confirmed to be that expected from the design values of the slow wave structure. Measurements in the vacuum region showed that power in electromagnetic modes, e1ther directly launched or parametrically produced, was negligibly small. 


\section{POWER BALANCE}

A key objective of these experiments was to determine the power balance during rf heating. In particular, since several times the ohmic power could be applied to the plasma with no significant increase in the central electron temperature, an understanding of the power balance is necessary to assess the potential of this heating scheme. Despite this lack of increase in the bulk plasma temperatures (or densities), there was ample evidence of non-thermal effects in both the ion and electron distributions. Distinctly different macroscopic phenomena were observed at the opposite extremes of the $n_{\|}$values studied. Moreover, measurements of the radiated power, the power in $\mathrm{rf}$ waves in the edge region, and absolute power in the charge exchange neutrals indicated the major power loss was not via these mechanisms.

Strong evidence that the rf power was transferred to particles in the plasma interior was obtained from measurements of the charged particle energy lost to the wall taken with a thermocouple probe. Figure 2 shows the temperature increase of the thermocouple probe as a function of the total energy input, rf and ohmic, for several spatial locations. The thermocouple probe is, of course, sensitive to incident particle energy. Particularly noteworthy here is linearity of the thermocouple response to applied energy, be it ohmic or rf energy. A slight deviation from the linear behavior is apparent for the highest 
rf power levels when the probe is inserted $4.4 \mathrm{~cm}$ from the 1 imiter. These results, coupled with the lack of significant power loss measured in other channels, are evidence that the rf energy was coupled to plasma particles within the the central $10 \mathrm{~cm}$ of the plasma and carried out by the charged particles. This thermocouple measurement does not constitute by itself a definitive result since cven in the ohmic case the probe samples only a fraction of the total power to the limiter and wa11; so if the rf energy is carried out by more energetic particles than typical. of the ohmic case a larger temperature increase could be obtained due to sampling a larger fraction of the rf-related power. However, the result was the same even when a shield was used to eliminate energetic electrons. Assuming the $\mathrm{rf}$ power is deposited in the central region and is lost in a similar manner as the ohmic input power, then the rf power can be accounted for fully. This measurement is significant not only bccauee it accounts for the $\dot{r f}$ power, but also because it eliminates stochastic heating in the electric field in front of the antenna as the major power drain. If in this model the rf power is coupled to plasma particles and then carried to the plasma edge where it is lost, it remains to determine which species, electrons or ions, is the loss channe1. The evidence for both species is presented below, but insufficient information exists to make a definitive choice.

The behavior of electron transport in tokamaks is poorly understood as evidenced by the lack of fundamental understanding of anomalous electron energy transport (reference 4). The complexity of the 
electron response to the application of $\mathrm{rf}$ power was apparent in the course of these experiments, and understanding the electron behavior was complicated by the presence of non-thermal effects which prevent simple interpretation of some of the diagnostics for bulk electron temperature.

The electron response to the $\mathrm{rf}$ power was sensitive to the value of $n_{\|}$of the launched wave. For the lowest $n_{\|}$, application of $r f$ power at low density resulted in significant increases in the plasma conductivity along with evidence of non-thermal electrons from increases in the second and third harmonic synchrotron emissions and the soft $x$-ray PIN diode signals. Yet the Thomson scattering measurements did not show any significant bulk electron temperature increase for these low $\mathbf{n}_{\|}$ cases. At the other extreme, the application of $r f$ power at $n_{\|}=28$ did not show any macroscopic changes. Central electron bulk temperature increases were only observed with $n_{\|}$of $16^{*}$ and 22 . These central increases were sporadic and could not always be readily reproduced, athough certain 'necessary but not sufficient' conditions were identified.

Significant temperature increase in the electron bulk was well documented on three statistically significant data sets. The three cases include two at $\mathrm{n}_{\|}=16 *$ and one with $\mathrm{n}_{\|}=22$. The two $\mathrm{n}_{\|}=16$ * cases include an extensive earlier set $n_{\|}=16 * a$. with only Thomson scattering measurements of $T_{e o}$ available and a later set $n_{\|}=16^{*} b$ where the Thomson scattering observations are supported by measurements made using SXR energy spectrum analysis. For these three cases $\mathrm{n}_{\mathrm{eo}} \simeq 8 \times 10^{12} \mathrm{~cm}^{-3}$, and the central electron temperature without $\mathrm{rf}$ 
was such that $v / l / v_{e o} \simeq 1.9$. All three of the instances of increases in the central temperature occurred under relatively clean and stable discharge conditions. From both bolometer measurements and spectroscopic studies, impurity influx upon the application of rf was not significant.

The central temperature increase was relatively modest remaining below

$$
\frac{\Delta \mathrm{T}_{\text {eo }}}{\mathrm{T}_{\text {eo }}}\left(\frac{\mathrm{P}_{\mathrm{RF}}}{\mathrm{P}_{\mathrm{OH}}}\right)^{-1} \leqslant 0.3
$$

The maximum central temperature increase observed was $0.6 \mathrm{~T}_{\text {eo }}$. The electron density was not significantly changed in these cases. In the $n_{\|}=22$ and $n_{\|}=16^{*} b$ cases no measureable change occurred. In the $\mathbf{n}_{\|}=16 * a$ case a small $(\sim 7 \%)$ decrease in electron density occurred.

Instances of central electron temperature increases with $\mathbf{n}_{\|}=22$ were recorded with Thomson scattering at relatively low initial central temperature and density $\left(120 \mathrm{eV} \leqslant \mathrm{T}_{\mathrm{e}} \leqslant 180 \mathrm{eV}\right.$ and $\left.6 \times 10^{12} \mathrm{~cm}^{-3} \leqslant \mathrm{n}_{\mathrm{e}} \leqslant 9 \times 10^{12} \mathrm{~cm}^{-3}\right)$. These temperatures are about as low as can readily be produced and diagnosed in Doublet IIA. For a temperature $\mathrm{T}_{\text {eo }}=150 \mathrm{eV}$, the ratio of the phase to thermal velocities is $\mathrm{vll} / \mathrm{v}_{\text {eo }}=1.9$. The most complete documentation of central bulk electron temperature increases were observed using rf power at $n_{\|}=16^{*}$, with the Thomson scattering measurements supplemented by the soft $x$-ray energy analysis. The temperature on similar shots with no rf power applied was about $250 \mathrm{eV}$, so that again $\mathrm{v}_{\|} / \mathrm{v}_{\text {eo }} \simeq 1.9$. Spatial scans of 
the electron temperature with the Thomson scattering were not available.

The conductance change observed at high rf power $\left(\mathrm{P}_{\mathrm{RF}} / \mathrm{P}_{\mathrm{OH}}>1\right)$ were less than expected from spatially uniform heating with Spitzer conductivity. This seems to indicate the heating is spatially localized, but anisotropic modifications of the electron distribution function can result in an enhancement of the number of trapped electrons which could limit the conductivity increase.

The time histories of the second harmonic synchrotron emission near the center of the discharge rise smoothly in $\sim 1 \mathrm{msec}$, remain constant throughout the rf period and fall to the pre-rf level. These signals might readily be mistaken as evidence of a thermal electron distribution in the absence of additional information.

Spatial scans show only modest increases in the center of the plasma, and much larger ( $10 \mathrm{x}$ ) increases at frequencies corresponding to the outside of the plasma. These large peaks could be due to trapped particles. In addition, one must consider that if the energy of the electrons is sufficiently high, their radiation is mass shifted to lower frequency, and thus their apparent location moves radially outward. Evidence of a shift of up to $10 \mathrm{~cm}$, corresponding to $60 \mathrm{keV}$ electrons, was observed.

Considerable progress was made in understanding the response of the electrons to the rf power when a soft $x$-ray energy spectrometer system was available during the last few months of the experiment, although these investigations were restricted primarily to $n_{\|}=16^{*}$. A 
typical soft $x$-ray spectrum is shown in Figure $3 \mathrm{~A}$. The measured distributions can be characterized by up to three distinct temperatures, the bulk temperature $T_{0}$ and two tails $T_{1}(\sim 4 \mathrm{keV})$ and $T_{2}(\sim 12 \mathrm{keV})$. Figure $3 \mathrm{~B}$ shows the electron temperature as a function of time from both the Thomson scattering (central) and soft $x$-ray (vertical chord) measurements. The sof $t \mathrm{x}$-ray measurement averages over a $5 \mathrm{msec}$ time interval. A surprising feature of this measurement is the continued increase in central electron temperature af ter the rf power is turned of $f$.

The time constants for the central bulk electron temperature increase can be associated with those observed for the formation and decay of the energetic electron tail component $\mathrm{T}_{2}$ observed in the soft $x$-ray spectrum. The presence of the tail seemed to be necessary but not sufficient to obtain a significant increase in the central bulk temperacure. This componeul Lypically had a characteriatic tompcrature of $\mathrm{T}_{2}>10 \mathrm{keV}$ and a fractional density of roughly $\mathrm{n}_{2} / \mathrm{n}_{0}=0.001$. This component decayed with a time constant of about $5 \mathrm{msec}$. The presence of this component could not be explicitly related to any one variable within the limited data set avallable.

The tail component $T_{1}$ was always present. Its temperature increases roughly as the square root of the power for the range of density available $\left(6-8 \times 10^{12} \mathrm{~cm}^{-3}\right)$, and the energy, nT, in this tail increases linearly with power. The fractional density of the tail was estimated from the soft $x$-ray measurements to be $n_{1} / n_{2}=2$ for a power level of $150 \mathrm{~kW}$, based on the assumptions that the tail is isotropic and its spatial distribution is identical to that of the bulk. This 
estimate is also subject to errors in the exact calculation of the coefficients. A limitation of the soft $x$-ray system for non-thermal distributions arises due to the viewing cone of the detector which does not see electrons magnetically trapped with high perpendicular energies; other diagnostics such as the synchrotron emission at the second harmonic show evidence of such a component as was discussed above.

The magnitude of the electron tails did not increase in the presence of a tail of lesser temperature in the distribution. Evidence from the decay of the synchrotron emission signal suggests the energy is in the perpendicular component and is not strongly influenced by the ohmic driving field.

The tail temperature $T_{1}$ was directly measured for $n_{\|}=16$ and $n_{\|}=14$ at $800 \mathrm{MHz}$ in addition to the major body of the data at $\mathbf{n}_{\|}=16 *(915 \mathrm{MHz})$. The tail temperature increases strong1y with the wave velocity $\left(v_{\|}{ }^{-6}\right)$, and since the calculated fractional density $n_{1} / n_{0}$ is essentially constant for these cases, the tail energy likewise increases. The tail temperature, and thus energy, doubles when $n_{\|}$is decreased from 16 to 14 at $800 \mathrm{MHz}$. No examples of the presence of a tail $\mathrm{T}_{2}$ were observed in the limited data set at $800 \mathrm{MHz}$. The temperature $T_{1}$ increased rapidly with the frequency of the applied wave $\left(f^{6}\right)$. While this dependence is supported qualitatively by other diagnostics, earlier qualitative data would seem to indicate no frequency dependence. 
There was also no evidence of any significant accumulation of the tail heating effects when power from the several sources was applied simultaneously. With a total power of $360 \mathrm{~kW}$ was applied ( $140 \mathrm{~kW}$ at $\mathrm{n}_{\|}=16 *, 100 \mathrm{~kW}$ at $\mathrm{n}_{\|}=14$, and $120 \mathrm{~kW}$ at $\left.\mathrm{n}_{\|}=16\right)$, there was no additional contribution to the tail components that was measurable beyond that observed for $140 \mathrm{~kW}$ of power at $n_{\|}=16 *$ applied alone.

The accumulated data on the electron behavior during rf heating shows evidence of strong interaction with the lower hybrid waves. However, attempts to quantify the energy in the electron distribution does not readily balance with the rf power applied.

The production of a significant hot ion component as determined by the charge exchange neutral analyzer was an ubiquitous feature of the application of rf power to the plasma. Parametric decay spectra were also observed on the limiter floating potential for all cases examined.

Detailed measurements of the Ion temperature were made using the charge exchange diagnostic. For example, the application of $185 \mathrm{~kW}$ of rf power at $n_{\|}=16$ * to a plasma with a bulk ion temperature of $134 \mathrm{eV}$ before the rf power is applied, fitted temperature is $239 \mathrm{eV}$ with the rf power applied. The ions sampled could originate anywhere along the vertical central chord, and the temperatures are inferred from ions with energies greater than $600 \mathrm{eV}$. Although the charge exchange signals appear Maxwellian, other measurements show the temperature increase with $\mathrm{rf}$ power is not thermal and is related to the formation of an Ion tail distribution. 
The observed rise and fall times of the signals from the individual channels of the charge exchange analyzer are very short $(\sim 50 \mu \mathrm{sec})$. These time constants decrease with energy. There is no evidence of a significant long lived decay component to indicate the presence of a bulk increase due to the thermalization of the tail. In fact, the presence of a measureable bulk increase in the ion temperature after the $r f$ power was turned of $f$ was never detected in any interval. Because of the rapid rise time and the finite amount of power available less than $10 \%$ of the ions can be involved.

The measured ion tail temperature increased linearly with applied rf power (Figure 4). The zero power intercept of the extrapolated temperature was found to be equal to the thermal temperature with no $r f$ power applied. The linear power dependence held for all antennas tested $\left(n_{\|}=16,16^{*}, 19\right)$ over a wide range of plasma parameters. The extrapolation of the linear power dependence to the central thermal temperature without rf power applied would seem to indicate that the heating occurs in the central region. However, since this diagnostic is sensitive to ions which have a turning points along the central vertical chord, a distortion of the ion velocity distribution function even near the edge could produce the measured charge exchange signals.

The rate of increase of the ion tail temperature observed with rf power applied varied widely amongst the antennas studied, but was relatively constant from shot to shot for the various antennas. The antenna efficiency cannot be systematically related to the value of $n_{\|}$, in fact the widest variation occurred between two mechanically identical antennas installed symmetrically above and below the discharge 
column. The relative efficiency of these two antennas reversed with the toroidal field direction. The asymmetry was not simply related to gradient $B$ drifts since the antennas at the other toroidal azimuths exhibited the opposite dependence on toroidal field direction. There is no corresponding asymmetry in the interaction of these antennas with the electrons.

The intercepts of the charge exchange data indicate the product of the neutral and ion densities decreases by approximately a factor of two with the rf power on. Since the available power is only sufficient to heat 10 percent of the bulk ions to the measured temperature, this is evidence that either the hot ion distribution is not isotropic but heated selectively in the transverse plane or the hot ions are relatively near the edge of the plasma so that the neutral density is increased, or both. The thermocouple probe measurements discussed earlier and the linear extrapolation of the hot ion temperature with power to the central ion temperature at low power indicate the heated ions if they account for significant power loss are not near the edge. 
IV. CONCLUSION

The evidence accumulated during the course of the Doublet IIA experiments indicates the energy in lower hybrid waves is efficiently coupled to the plasma and propagated to the central region of the discharge. The only evidence of non-linear coupling or propagation is the existence of parametric decay spectra at the plasma edge. The absorption of wave energy, however, appears to be strongly influenced by non-linear mechanisms; perpendicular electron tails with energy much larger than expected from quasi-1inear Landau damping theory are observed as well as ion tails. The lack of spatial information and quantitative measurements of the energy in these tail components prevents a definitive modeling of the heating, but bulk heating of either ions or electrons is prevented by the loss of the heated species before thermalization can occur. Evidence exists for both electrons and ions as the major loss channel, but unresolved questions remain for either interpretation.

The power balance might easily be satisfied by the ions. As indicated, less than $10 \%$ of the ions in the tail can account for all of the power. The thermocouple probe evidence that most of the energy is deposited in the central $10 \mathrm{~cm}$ of the plasma would require, according to accepted theory, that the energy be transmitted to the central region by parametric decay waves with lower $\mathrm{n}_{\|}$since under some conditions where the ion tails were observed the launched waves should 
be strongly Landau damped on the electrons. The most likely loss process for the ions is direct particle loss to the limiters due to large banana orbits. For the energies observed with the charge exchange spectrometer the maximum orbit is about $7 \mathrm{~cm}$, and the bounce time for these ions is in reasonable agreement with the observed loss time. The absolute power lost through charge exchange is small (few $\mathrm{kW}$ ), so this is not a major loss channel. Although the ions could be the major energy loss channel it is also possible that the observed effects are due to only a small fraction of the ions since the charge exchange analyzer is sensitive to only a small portion of ion velocity space. The observed asymetry in antenna efficiency indicated spatially localized effects which supports this view.

The energy accounting in the electron channel does not readily balance the applied rf power. Estimates from the soft x-ray measuremenls accuunt for less than $25 \%$ of the applied power, but good . correlation was documented between central bulk electron temperature increases and the decay of the most energetic electron tail $\left(\mathrm{T}_{2}\right)$. Also, the energy in the lower temperature electron tail $n_{1} T_{1}$, increased linearly with rf power. The central bulk electron temperature inrease was only observed in plasmas with low impurity levels and when the ratio of the Landau velocity to the central thermal velocity was about 1.9. This approximately velocity ratio is approximately that expected if quasi-linear Landau damping is the operative mechanism. However, these conditions appear necessary but not sufficient for the heating. A major uncertainty in the power balance arises due to the limited 
viewing cone of the soft $x$-ray detector, since the synchrotron emission shows evidence of even more energetic electrons but quantitative information is lacking. Interpretation of the synchrotron emission due to non-thermal particles is very complicated and has not been done, but rough estimates indicate a substantial fraction of the thermal energy could be stored in the $60 \mathrm{keV}$ magnetically trapped electrons. If significant energy is stored in the energetic electrons relative to that stored in the plasma, then radial column shifts are expected which are not observed. Consequently, to account for the rf power in energetic electrons, their confinement must be considerably worse than the bulk electrons. However, the measured decay time of the synchrotron emission signals for the $60 \mathrm{keV}$ component is approximately $1 \mathrm{msec}$ which is comparable to the bulk decay times.

The loss of energetic electrons is consistent with recent theoretical understanding of Alcator A transport data (reference 5) and could represent a serious impediment for utilization of: Landau damping of lower hybrid waves by electrons as a tokamak heating technique. Experiments in higher density plasmas may impede the tail formation, and should also allow better thermalization with the bulk electrons due both to more rapid thermalization and the better bulk electron confinement.

We acknowledge useful discussions with Professor Abe Bers of Massachusetts Institute of Technology on the interpretation of these experiments. Also, we are indebted to Doug Remsen for the trouble-free 
operation of the rf power systems, to Earl Heckman for data acquisition assistance, to Randy Hager and his staff for antenna fabrication, and especially to the operating staff of Doublet IIA. 
TABLE 1

DOUBLET IIA OPERATING PARAMETERS

\begin{tabular}{|c|c|}
\hline Major radius $\mathrm{R}(\mathrm{cm})$ & 66 \\
\hline Minor radius a (cm) & 15 \\
\hline Toroidal magnetic field $\mathrm{B}_{\mathrm{T}}(\mathrm{kG})$ & 7.8 \\
\hline Operating gas & $\mathrm{H}_{2}, \mathrm{D}_{2}$ \\
\hline Plasma current $I_{p}(k A)$ & $20-40$ \\
\hline Ohmic power $(\mathrm{kW})$ & $50-120$ \\
\hline Central electron temperature $\mathrm{T}_{\text {eo }}$ & $150-350$ \\
\hline Average electron density $\dot{n}_{e}\left(x^{1} 10^{13} \mathrm{cn}^{-3}\right)$ & $0.5-1.5$ \\
\hline$Z_{\text {eff }}$ & $1-5$ \\
\hline
\end{tabular}




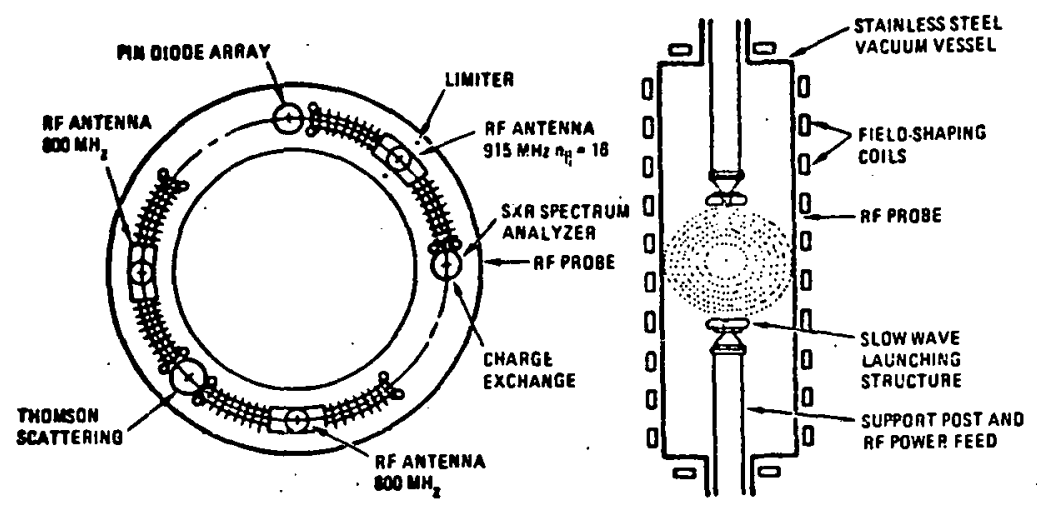

Figure 1
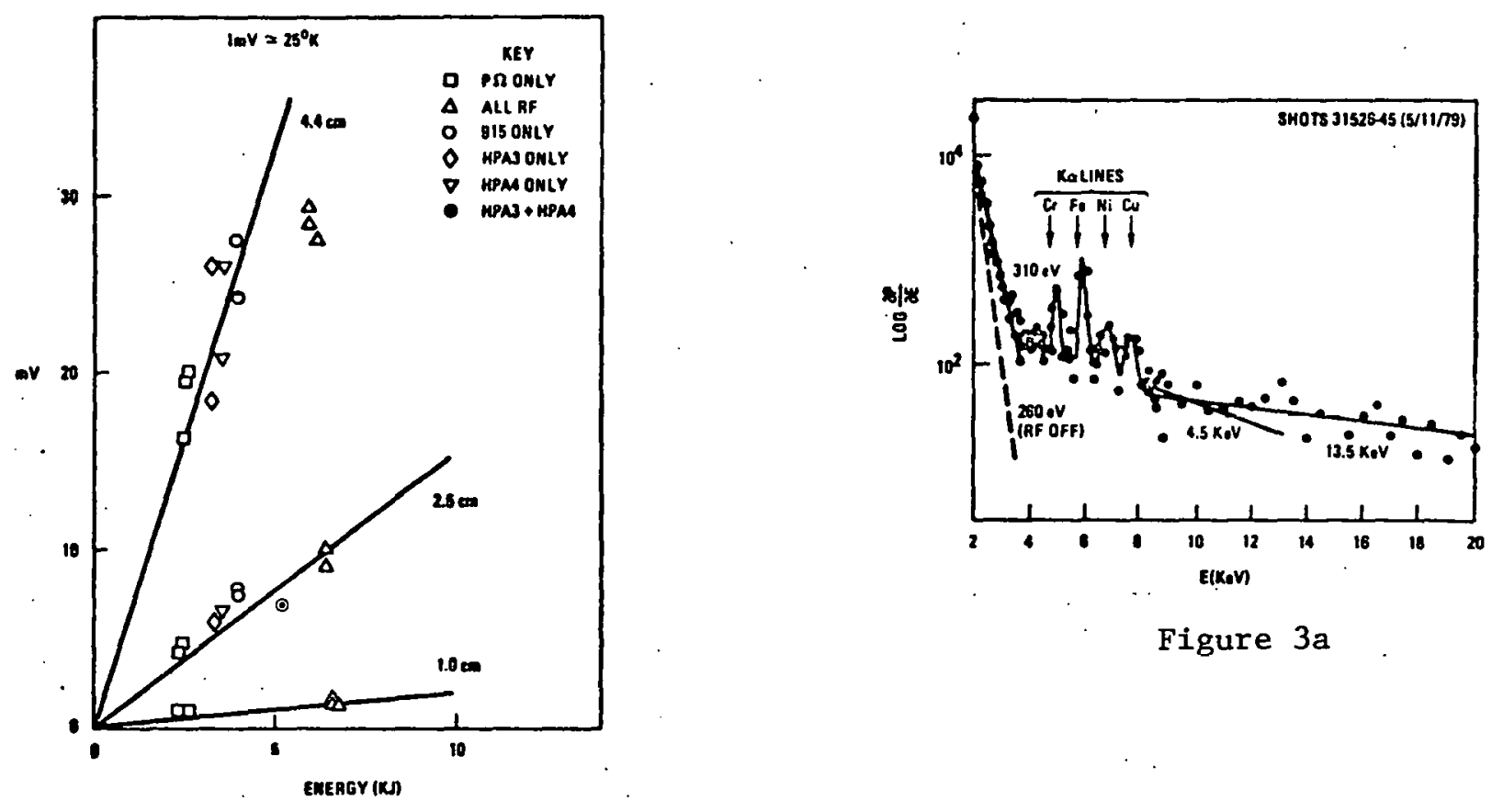

Figure 3a

Figure 2

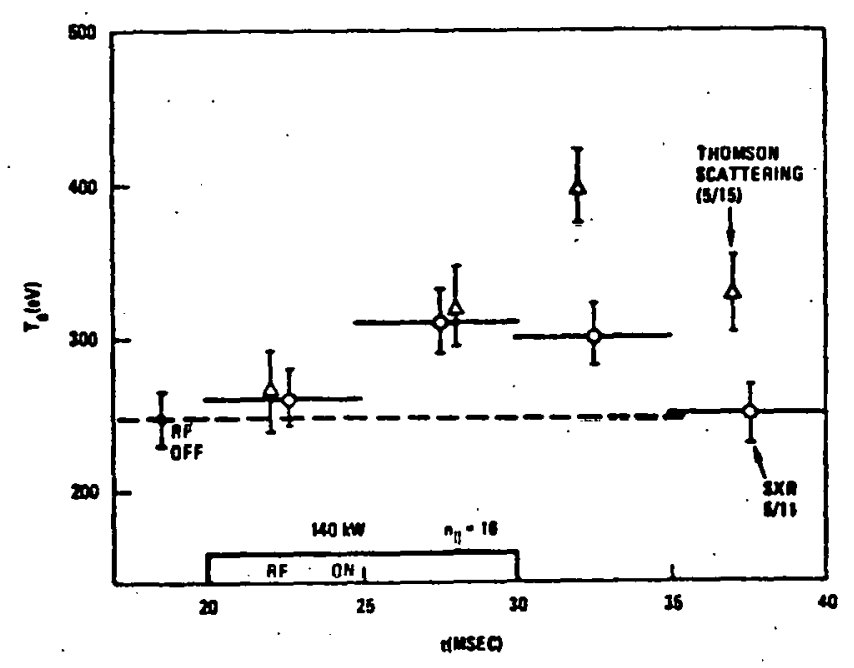

Figure $3 b$

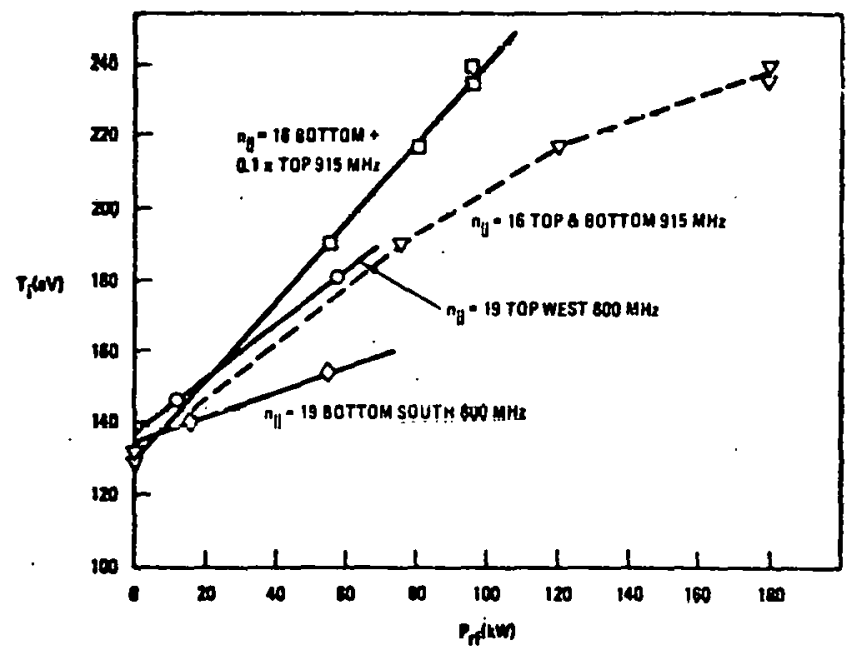

Figure 4 


\section{References:}

1. J.L. Luxon, et.al. to be published.

2. R.K. Fisher, et.al. Phys. Rev. Lett., $\underline{39}$ 622(1977)

3. C. Moeller, and Doublet II Group proceedings of the Third Topical Conference on Radio Frequency Plasma Heating. Pasadena (1978) paper A4. See also V.S. Chan, C. Moeller and R.W. Gould, paper B2

4. R.A. Parker, Bull. Am. Phys. Soc: 20, 1392(1975)

5. Kim Molvig, John E. Rice, Miloskav S. Tekula, Phys. Rev. Lett. $\underline{41} 1240(1978)$.

\section{Figure Captions:}

Figure 1. Top and cross-section views of Doublet IIA showing slowwave structures.

Figure 2. Thermocouple probe signal versus total applied energy (RF plus ohmic) with $1 \mathrm{~cm}, 2.5 \mathrm{~cm}$, and $4.4 \mathrm{~cm}$ from the limiter location.

Figure 3a. Typical soft $x$-ray energy spectrum (observed along a vertical chord) with $\mathrm{rf}$ power applied. $\left(140 \mathrm{~kW} \mathrm{n}_{\|}=16 *\right)$

Figure 3b. Time dependence of the electron temperature as measured by Thomson scattering and soft $x$-ray energy analysis with $140 \mathrm{~kW}$ of $\mathrm{rf}$ power at $\mathrm{n}_{\|}=16 *$.

Figure 4. Ion 'tall' temperature as a function of applied $r f$ power for various $n_{\|}$values and antenna locations. The upper solid line demonstrates that the efficiency of the top $\mathbf{n}_{\|}=16 *$ antenna for this toroidal field direction (see text) is about $10 \%$ relative to the bottom antenna.

**Work supported by Department of Energy Contract DE-AT03-7651011 


\section{EXTERNAL DISTRIBUTION}

Dr. S. Bernabei

Dr. Abe Bers

Dr. Ron Blanken

Dr. John Clarke

Dr. Anne Davies

Dr. A. England

Dr. K. Fowler

Dr. Bill Hooke

Mr. Edwin E. Kintner
Dr. George Moralis

Dr. Bob Motley

Dr. Mel Oakes

Dr. Horst Pacher

Dr. R. S. Pease

Dr. M. Porkolab

Dr. J. Shearer

Dr. Jack Schuss

Dr. John Willis 